\title{
Sclerostin Antibody Prevented Progressive Bone Loss in Combined Ovariectomized and Concurrent Functional Disuse
}

Dongye Zhang, M.E./M.S. ${ }^{1}$, Minyi Hu, Ph.D. ${ }^{1}$, Timothy Chu ${ }^{1}$, Liangjun Lin, Ph.D. ${ }^{1}$, Jingyu Wang, M.S. ${ }^{1}$, Xiaodong Li, Ph.D. ${ }^{2}$, Hua Zhu Ke, M.D. ${ }^{3}$, Yi-Xian Qin, Ph.D. ${ }^{1 \#}$

${ }^{1}$ Dept. of Biomedical Engineering, Stony Brook University, Stony Brook, NY 11794-5281

${ }^{2}$ Dept. of Metabolic Disorders, Amgen, Inc., Thousand Oaks, CA

${ }^{3}$ UCB Pharma, Slough, UK

\author{
\# Corresponding Author \\ Yi-Xian Qin, Ph.D. \\ Address: Dept. of Biomedical Engineering \\ State University of New York at Stony Brook \\ 215 Bioengineering Bldg.
}

Stony Brook, NY 11794-5281

Tel: 631-632-1481

Email: yi-xian.qin@stonybrook.edu

No supplemental data has been included with the submission 


\section{Disclosure}

Xiaodong Li and Hua Zhu Ke has/had employment at Amgen, Inc. 
Key Words: severe osteoporosis; mechanical unloading; post-menopause; sclerostin antibody; histomorphometry 


\section{Introduction}

Osteoporosis is characterized by compromised bone mass and microarchitectural deterioration [1-3]. Currently, it is estimated to affect more than 10 million Americans, cause about 1.5 million osteoporotic fractures each year and put an estimated 44 million Americans at risk, which exerts an extensive financial burden on the healthcare system with a direct annual medical cost of around 20 billion dollars[4]. With the growth of aging population, the incidence of osteoporosis and prevalence of osteoporotic fractures is expected to increase dramatically [5$8]$.

Significant bone loss related to mechanical disuse or loss of weight bearing in prolonged bed-ridden patients represents a critical issue [9-11]. Numerous biochemical parameters showed uncoupling between bone resorption and formation markers as well as an increased number of osteoclasts and enlarged resorption cavities in histomorphometric studies in prolonged immobilized subjects $[10,12,13]$. A considerable imbalance between bone formation and resorption induced by abrupt decline of ovarian production of estrogen in post-menopausal women represents another primary cause of the condition of osteoporosis [14]. Women over 50 years of age have also been reported as the most sedentary group of the adult populations [15]. Lack of mechanical stress and physical loading deteriorates bone integrity as exercise was proved to be beneficial to preserve bone mineral density (BMD) and prevent bone loss conditions $[16,17]$. The two risk factors above expose elderly female to greater risk of osteoporosis and fractures. Therefore, It is highly clinically relevant to examine the severe osteoporosis condition due to estrogen deficiency accompanied by concurrent mechanical unloading where more rapid and devastating bone mass deterioration is present, in conditions such as post-menopausal women who are confined to bed after the onset of stroke or accidents 
$[10,18]$ and a better understanding of the interactions of the two risk factors may help lead to a better prevention and treatment of the disease.

Sclerostin, an osteocyte-expressed glycoprotein encoded by SOST gene, acts as a negative regulator of bone formation by antagonizing Wnt/ $\beta$ catenin signaling $[2,19-22]$. Both extended immobilization and condition of post-menopause has been related to an increase of sclerostin serum levels $[10,22,23]$. Patients with homozygous inactivation mutation of SOST gene develops sclerosteosis, a rare genetic disorder characterized by undetectable level of serum sclerostin, very high bone mass phenotype and nerve entrapment due to excessive bone formation. Individuals with heterozygous carriers for the mutation had increased bone mineral density and lower risk of fractures. This discovery has led to the hypothesis that sclerostin neutralizing agents might mimic the heterozygous carrier condition and be efficacious in reversing bone loss in osteoporosis [24-26]. Targeted deletion of SOST gene in mice resulted in increased bone formation, bone mass and bone strength whereas overexpression of SOST leads to an osteoporotic phenotype in mice $[11,19,21,27]$.

It has been the emphasis of multiple recent studies to target Wnt/ $\beta$ catenin signaling pathway to promote bone formation $[11,19,28]$. Using monoclonal antibody to inhibit sclerostin seems to be a very promising strategy in that SOST is almost exclusively expressed in osteocytes $[29,30]$. Sclerostin- neutralizing monoclonal antibodies (Scl-Ab) has been tested in disuseinduced and OVX-induced osteoporosis animal models. In a right hind-limb-immobilization female rat model, histomorphometric data showed that Scl-Ab mediated blockade of sclerostin dramatically enhanced bone formation with an increased bone mass and reduced bone resorption [31]. In an aged OVX female rat model simulating postmenopausal osteoporosis, twice per week subcutaneous Scl-Ab injection at $25 \mathrm{mg} / \mathrm{kg}$ was administered for 5 weeks. Micro-CT and 
histomorphometry data exhibited significant increases in bone formation and bone mass comparing to the placebo groups. These changes together with improved stiffness and maximum load data obtained from destructive four-point bending tests indicate an improved skeletal strength [2].

All the evidences above consolidate the notion that Scl-Ab -mediated blockade of sclerostin represents a promising therapeutic approach for the treatment of osteoporosis induced by either immobilization or post-menopause. However, little is known about the effect of prolonged and severe osteopenia in bone loss induced by concurrent mechanical unloading and OVX. The efficacy of Scl-Ab in rats suffered from severe osteoporosis with presence of both prolonged immobilization and estrogen deficiency is yet to be determined. The aim of the study is to evaluate the effect of $\mathrm{Scl}-\mathrm{Ab}$ in a severe progressive bone loss from combined estrogen deficiency and functional immobilization, such as under OVX with concurrent hindlimb suspension disuse in a rat model. Bone volume fraction, mechanical strength, bone formation rate, and plasma formation/resorption markers in such severe osteopenia and osteoporosis conditions were examined.

\section{Materials and Methods}

All experimental procedures with the described animal models were approved by the Institutional Animal Care and Use Committee (IACUC) at Stony Brook University. Animals

Seventy-seven 4-month-old female Sprague-Dawley rats (Charles River, Wilmington, MA) weighing an average of $327 \pm 35 \mathrm{~g}$ were purchased at 4-month of age and housed individually in a temperature-controlled animal facility with a 12:12 hour light:dark cycle at 
Stony Brook University. They were allowed free access to chow diet and water. Thirty-three rats that had undergone ovariectomy surgery and forty-four rats that had undergone sham surgery (Sham) 10 days before the arrival were subject to either functional disuse or free ambulance after 2 days of acclamation. Functional disuse was induced by HLS where the immobilization was realized by a tail harness device attached to tail that had been cleaned with $70 \%$ isopropanol and briefly coated with benzoin. The other end of the harness was attached to a swivel apparatus that was suspended from the roof of the cage. Due to an approximate $30^{\circ}$ head-down tilt, the hindlimbs of the animals were prevented from touching the ground of the cage at all time during the experiment, while free ambulance of forelimbs was allowed [32, 33]. All animals were checked twice a day to monitor their health conditions and their weights throughout the study.

\section{Scl-Ab treatment}

The animals were divided into 7 groups with $n=11$ per group: (1) Sham+Veh, (2)

Sham+HLS+Veh, (3) Sham+HLS+Scl-Ab, (4) OVX+Veh, (5) OVX+Scl-Ab, (6) OVX+HLS+Veh, (7) OVX+HLS+Scl-Ab. Group 1, 2, 4 and 6 were injected with PhosphateBuffered Saline (PBS) subcutaneously twice per week for 5 weeks while Group 3,5 and 7 were injected with a sclerostin monoclonal antibody (Scl-Ab; Amgen, Thousand Oak, CA, USA) at $25 \mathrm{mg} / \mathrm{kg}$ at the same frequency and duration $[34,35]$. The treatment of either vehicle or Scl-Ab started at the time that is approximately 2 weeks after sham or OVX surgery. The antibody was shipped to Stony Brook University on dry ice from Amgen and stored in $-80^{\circ} \mathrm{C}$ until thawed for making injection solution.

$\mu C T$ analysis

After five weeks of study, animals under isoflurane anesthesia were euthanized via cervical dislocation to ensure rapid euthanasia. Both right and left hind-limbs of rats from all the 
groups were harvested and preserved in $70 \% \mathrm{EtOH}$ at $-20{ }^{\circ} \mathrm{C}$. A high resolution $\mu \mathrm{CT}$ scanner ( $\mu$ CT-40, SCANCO Medical AG, Bassersdorf, Switzerland) was adopted for scanning distal portion of right femurs with a spatial resolution of $15 \mu \mathrm{m}$ and a specific Gaussian sigma, support and lower threshold of $0.5,1$ and 330 for defining analyzed objects and image smoothing purpose. One $750 \mu \mathrm{m}$ region of trabecular bone that was $300 \mu \mathrm{m}$ proximal to the epiphyseal plate of distal femur was chosen as the area of interest. Bone volume fraction (BV/TV, given as \%), trabecular thickness $(\mathrm{Tb} . \mathrm{Th}, \mathrm{mm})$ and structure model index (SMI) were evaluated for the trabecular region.

\section{Mechanical Testing}

Mechanical testing was performed by 4-point bending on the mid-shaft of right femur, which produces stress between two upper loading points while the lower two points serve as support. Servo hydraulic MTS 858 Mini Bionix frame (MTS Systems, Eden Prairie, MN, USA) was adopted to perform the bending. Specimens were placed on the frame horizontally with midshaft of the diaphysis facing to the notch in the same position and orientation in order to minimize the variation. The rate of displacement was set to be $1 \mathrm{~mm} / \mathrm{min}$ with a preload of $1 \mathrm{~N}$ and the bending was imposed on specimen continuously until fracture occurred [36]. The maximal load and the curve of load-displacement were monitored. Based on the loaddisplacement curve plotted, the maximum bending force applied until fracture was recorded as ultimate force $(\mathrm{N})$ and slope of the linear portion that represents material elasticity was used to calculate stiffness $(\mathrm{kN} / \mathrm{mm})$ [37].

\section{Histomorphometry}

All rats were subject to two subcutaneous calcein (Sigma-Aldrich Inc., St.Louis, MO, USA) injections at $15 \mathrm{mg} / \mathrm{kg} 3$ days and 13 days before euthanasia. After $\mu \mathrm{CT}$ scanning and 4 
point bending, the distal portions of right femurs were cut against fractures made by mechanical test and subsequently dissected into two equal-size halves along the sagittal plane by using low speed diamond wheel saw (South Bay Technology, Inc., San Clemente, CA, USA). The lateral sides of distal femurs were saved for future studies while the medial sides were dehydrated with isopropanol and used for histomorphometry. The samples were then infiltrated and embedded with a mixture of methyl methacrylate, di-butyl phthalate and benzoyl peroxide. After the media were cured, longitudinal slices were sectioned to $5 \mu \mathrm{m}$ using a Leica 2165 microtome (Leica, Wetzlar, Germany). The region of interest encompasses the trabecular area that was scanned by $\mu \mathrm{CT}$ system earlier. A section area of approximately $0.6 \mathrm{~mm}^{2}$ at metaphyseal area of the bone was examined by using the Osteomeasure software (OsteoMetrics Inc., Decatur, GA, USA) to trace the calcein label in the trabecular bone under fluorescent microscope. Histomorphometric bone volume fraction (aBV/TV, \%), trabecular thickness (aTb.Th, $\mu \mathrm{m})$, mineralizing surface/bone surface (MS/BS, \%), mineral apposition rate (MAR, um/day) and bone formation rate $\left(\mathrm{BRF} / \mathrm{BS}, \mu \mathrm{m}^{3} / \mu \mathrm{m}^{2} / \mathrm{yr}\right)$ were evaluated.

\section{ELISA analysis of resorption marker}

Blood samples were obtained from the caudal vein of animals under anesthesia at week 5 during sacrifice for tartrate-resistant acid phosphatase 5b (TRACP 5b) testing. All the samples were kept in $1.5 \mathrm{ml}$ Eppendorf tubes that had been pre-washed with heparin. Plasma samples were obtained by centrifuging the blood samples at a speed of 3000rpm at $4{ }^{\circ} \mathrm{C}$. Plasma aliquots were then stored at $-80^{\circ} \mathrm{C}$. Plasma bone turnover marker (BTM) level of TRACP $5 \mathrm{~b}$ were examined and quantified by adopting immunological assay (IDS Inc., Scottsdale, AZ). All testing was performed according to manufacturer's protocols. 


\section{Statistical analysis}

All the results were reported as mean \pm SD for all of the analysis. $\mu \mathrm{CT}$, histomorphometry and bone turnover marker data were all evaluated by using GraphPad Prism software (Version 5.01, GraphPad Software Inc., San Diego, CA, USA). One-way analysis of variance (ANOVA) with Tukey's pairwise multiple comparison tests were performed on all data from the distal femur metaphysis. The level of significant differences between two groups was identified by using a $p$ value of less than 0.05 .

\section{Results}

$\mu C T$

As shown in Figure 1a and 1b, $\mu \mathrm{CT}$ analysis of the metaphyseal region of distal femur revealed 5 weeks of mechanical unloading alone resulted in $29 \%$ loss in trabecular bone volume compared to sham control. Vehicle-treated OVX rats had a significant $71 \%$ decrease in BV/TV. OVX rats with concurrent HLS resulted in an even greater bone loss of $87 \%$, representing a severe bone loss condition. Scl-Ab treatment showed a counteractive effect on bone mass loss. $\mathrm{Scl}-\mathrm{Ab}$ treatment was associated with significantly greater BV/TV with an increase of $111 \%$ and $229 \%$ in HLS and OVX rats compared with respective control. Even greater increase in BV/TV (297\%) was found in OVX + HLS group (Figure. 1b), likely due to extremely low BV/TV in vehicle-treated OVX + HLS rats. Tb.Th did not change significantly in rats with either HLS alone or OVX alone, however, rats with concurrent OVX and HLS showed a significant decrease of Tb.Th (-26\%) (Figure. 1c). Scl-Ab administration significantly improved Tb.Th to similar extent in HLS, OVX and OVX+HLS rats compared with respective vehicle control (88\%, 87\% and $97 \%$ respectively). Structure model index (SMI) (Figure. 1d) was significantly greater in HLS alone group, OVX alone group as well as OVX+HLS group $(+73 \%,+129 \%$ and $+184 \%$, 
respectively) compared with sham control. In rats treated with Scl-Ab, SMI was significantly lower $(-396 \%,-159 \%$ and $-46 \%)$ in all three conditions compared with respective controls.

\section{Mechanical Testing}

Four-point bending test demonstrated that HLS alone or OVX+HLS were associated with significantly lower stiffness as compared with Sham controls (-23\% and -29\% respectively) (Figure. 2a). In Scl-Ab treated groups, stiffness was significantly greater in all 3 conditions $(+31 \%,+32 \%$ and $+57 \%$, respectively) compared with respective controls. Ultimate load was not significantly lower in HLS, OVX or OVX plus HLS as compared with Sham controls, but it was significantly greater in Scl-Ab-treated HLS or OVX rats as compared with respective vehicle controls and sham group (Figure. 2b).

\section{Histomorphometry}

Figure. $3 \mathrm{f}$ shows the representative images of calcein labeled trabecular bone. The data of histomorphometric analysis of distal femoral metaphysis trabecular bone is presented in Figure. 3a-3e. OVX alone had significant decrease of BV/TV at -36\% in comparison to sham control. HLS plus OVX showed greater bone loss (-71\%) than HLS alone and OVX alone. Scl-Ab treatment significantly preserved bone mass in all three conditions. Similar trend was observed in Tb.Th. These results are generally consistent with $\mu \mathrm{CT}$ analysis. MS/BS was significantly greater in three $\mathrm{Scl}-\mathrm{Ab}$ treated groups compared with respective controls. MAR and BFR/BS were significantly greater in Scl-Ab treated OVX rats with or without HLS compared with respective controls.

\section{ELISA analysis of resorption marker}

Bone turnover marker levels normalized to age match control are shown as percentage difference in Figure. 4. At the end of the study, plasma TRACP 5b level in the Sham+HLS+Scl- 
$\mathrm{Ab}$ rats were significantly reduced relative to that in the corresponding Sham $+\mathrm{HLS}+\mathrm{Veh}$ rats. TRACP $5 b$ level in OVX+Veh rats showed a decreasing trend as compared with that in the agematch control rats. Scl-Ab treatment in OVX animals failed to recover this trend. TRACP $5 b$ level in OVX+HLS+Veh rats at sacrifice was at similar level relative to that in the age-match control animals, while administration of Scl-Ab in OVX+HLS rats maintained this trend.

\section{Discussion}

We used concurrent mechanical unloading in addition to OVX to study the effect of severe bone loss. The HLS animals and OVX animals used in this study developed substantial bone loss condition. Remarkably, the HLS+OVX animals were significantly more osteoporotic resulting from the additive effect of concurrent mechanical unloading and ovariectomy with $87 \%$ of bone loss compared to age-match control group. In this study, Scl-Ab is proposed as a therapeutic bone anabolic agent for treatment of bone loss based on its effectiveness in previous bone loss animal studies and human clinical trials $[31,38]$. Beyond the fact that our data indicated that short-term administration (5-week) of Scl-Ab resulted in complete reversal of the bone loss condition induced by prolonged mechanical unloading or OVX surgery, bone mass and mechanical parameters were further increased to levels greater than age-match controls as also evidenced in previous studies [2]. Whereas in the severe osteoporosis model introduced by concurrent mechanical immobilization and deficiency of estrogen, Scl-Ab was able to partially mitigate the deterioration of trabecular bone architecture and morphology as well as maintain mechanical robustness. SMI, which measures trabecular concavity, serves as an indirect method to provide indication of plate- or rod-like trabecular bone microstructure and an important predictor of fracture [39]. The SMI value usually ranged from 0 to 3, where 0 stands for an ideal plate structure while 3 stands for and ideal rod structure. Values between 0 and 3 corresponds to 
trabecular structures with different ratios of rod and plate structure [40]. The data of SMI strongly suggested that by HLS alone, OVX alone or HLS+OVX, geometry of trabeculae shift towards a more rod-like structure, while subcutaneous injection of Scl-Ab totally reversed the trend in HLS alone group and OVX alone group. HLS+Scl-Ab showed an even more significant plate-like geometry compared to the age-match control group, suggesting a less vulnerability to fractures. In the OVX+HLS induced severe osteoporosis, Scl-Ab moderately alleviated the condition by shifting the trabeculae towards a more plate-like geometry.

In addition to the diminished bone volume fraction and trabecular thickness in the severe osteoporosis scenario, OVX + HLS rats also had a jeopardized mechanical property, such as lower stiffness and ultimate load, which might be accounted by the thinning of cortical shell and reduced cortical bone volume (data not shown). The considerable improvement of the related parameters by short term administration of Scl-Ab to OVX+HLS rats could be rendered noticeable gain in whole bone strength at femoral diaphysis. This finding is divergent from previous study by Tian et.al, who stated that the lag of cortical response is hard to overcome by a short term treatment of Scl-Ab for 4 weeks after they found insignificant cortical histomorphometry response. In depth morphological and structural cortical bone studies will be needed to further characterize the tissue level changes of cortical bone and provide an explanation for the changes of the mechanical property changes seen in both untreated and Scl$\mathrm{Ab}$ treated animals.

Histomorphometric analysis showed that 2D bone volume fraction as well as $2 \mathrm{D}$ trabecular thickness changed in parallel with $\mu \mathrm{CT}$ results. Trabecular bone volume and trabecular bone thickness were dramatically decreased in severe osteoporosis model. Scl-Ab treatment in severe osteoporosis scenario significantly mitigated bone mass loss condition and totally 
reversed the bone thinning by surpassing the original Tb.Th level. Similar to previous rat studies $[2,41], \mathrm{Scl}-\mathrm{Ab}$ treated groups showed significant increase in key bone formation indices (MS/BS, MAR and BFR/BS) as compared to respective control. The observed increases in MS/BS suggest that sclerostin neutralizing resulted in a more robust bone mineralizing activity on trabecular surface. BFR/BS is significantly elevated in HLS or OVX treated with Scl-Ab whereas HLS+OVX treated with Scl-Ab shares a similar trend. Not surprisingly, in parallel with the robust bone morphologic parameters, $\mathrm{BFR} / \mathrm{BS}$ in Scl-Ab treated animals is significantly elevated as compared to respective control, indicating an active bone matrix mineral deposition and osteoblast activity. In previous studies, compared to the consistent effect of $\mathrm{Scl}-\mathrm{Ab}$ on osteoblast activities, the effect of Scl-Ab on bone resorption remains inconsistent (Ke, et al., Endocrine Review, 2012).

TRACP 5b, mainly produced by osteoclast and abundant in osteoclast cytoplasm [42], is well known as a bone resorption marker. In our present study, with 5 weeks administration of Scl-Ab, the plasma TRACP $5 \mathrm{~b}$ value significantly decreased in Sham+HLS rats, indicating Scl-Ab preserved bone volume via both pro-anabolic and anti-catabolic responses in mechanical unloaded rats. This result is supported by previous studies, where Shahnazari, et al. showed that Scl-Ab maintained the bone osteoclast surface while reduced serum concentration of TRACP $5 b$ in mechanically unloaded animals [11]. There was no significant change of TRACP 5b value in OVX alone animals regardless of the treatment. As the plasma TRACP $5 \mathrm{~b}$ levels is basically a reflection of osteoclast numbers rather than osteoclast activity [43, 44], the insignificant decrease of TRACP $5 \mathrm{~b}$ in OVX+Veh rats is thought to be due to the significant loss of bone volume as evidenced in micro CT and histomorphometry. In a similar long term study of Scl-Ab using OVX model, Li X et al. observed that in OVX animals, the TRACP 5b level had a decreasing 
trend as compared with Sham controls at week 6 [45]. In the OVX+HLS+Veh animals, TRACP $5 b$ level showed a slight decrease as compared to age-match control rats. This result was supported by an earlier study using the combination of OVX and concurrent mechanical unloading via sciatic neurectomy [46], in which Sun et al. observed a significantly lower serum TRACP 5 b level at week 2 following OVX surgery as compared with control group. However by week 4, the TRACP 5b level trended back to normal control level, which is thought to be correlated with the non-persistent bone loss profiles between Week 2 and 4 [47]. Interestingly, Scl-Ab administration for 5 weeks in OVX+HLS rats did not exert significant changes on TRACP $5 \mathrm{~b}$ level, indicating the osteoclast number was not increased despite the significant increase in BV/TV as evidenced by micro $\mathrm{CT}$ and histomorphometry data, which may imply the role of Scl-Ab in inhibiting osteoclast activity by suppressing osteoclast number in concurrent mechanical unloading and estrogen deficient rat model. Histomorphometry analysis of total number of osteoclasts by TRAP staining will be needed in the future to further verify this hypothesis. It is also possible that Scl-Ab may regulate osteoclast activities due to its ability to antagonize sclerostin, which has been reported to be able to increase osteoclast recruitment and activity $[48,49]$.

The mechanism of action and regulation of sclerostin as a negative regulator of bone formation is yet to be fully understood [50]. It is currently believed that sclerostin, an osteocytes exclusive glycoprotein, blocks the activation of Wnt signaling pathway by binding to lowdensity lipoprotein receptor-related proteins 5 and 6 (LRP5 and LRP6) that mediate the regulation of Wnt signaling [20,51], which in turn interferes the Wnt signaling by antagonizing the survival and promotional effects on mesenchymal stem cells of the osteoblastic lineage [22, $52,53]$. Numerous factors have been associated with SOST expression by osteocytes as well as 
its serum level, among which bone mechanical loading is suggested to be one crucial regulator. Osteocytes have been recently suggested to be the proximate cell population that is responsible for mechanotransduction as targeted ablation of osteocytes resulted in osteoporosis with mechanotransduction dysfunction [47]. Absence of mechanical loading by immobilization results in higher SOST levels and down regulation of Wnt signaling, thus directly links to disuse osteoporosis. Sex steroid hormone, especially estrogen, is another important relevant regulator of SOST level. Post-menopausal women have higher serum sclerostin level compared to premenopausal women. In post-menopausal women, sclerostin level is inversely related to the free estrogen index (FEI), which suggests that sclerostin level is directly suppressed by estrogens [54, 55]. However mechanism of the regulation and a complete characterization of the pathway are still under continuous study.

In summary, this 5-week study demonstrated that both lack of mechanical stimulation and OVX resulted in significant bone loss in metaphyseal region and weakened mechanical properties in mid-shaft of the femur whereas OVX with concurrent mechanical unloading lead to more advanced trabecular microarchitecture deterioration as well as further compromised mechanical properties. With short-term pharmacologic inhibition of sclerostin by using neutralizing Scl-Ab, bone mass and strength were significantly improved and normalized to or beyond age-match control level. Scl-Ab prevented trabecular bone loss in HLS-induced, OVXinduced as well as HLS+OVX induced osteoporosis. These results suggest that monoclonal sclerostin-neutralizing antibody represents a promising anabolic therapeutic approach for severe osteoporosis induced by estrogen deficiency with concurrent mechanical unloading. Potential application of the agent may have a great impact on elderly female population with reduced physical activities. 


\section{Acknowledgments}

This work is kindly supported by the National Institute of Health (R01 AR52379 and R01 AR61821), the US Army Medical Research and Materiel Command, and by the Amgen, Inc. The authors thank Amgen, Inc. for providing Scl-Ab. The authors would also like to thank all the members in the Orthopaedic Bioengineering Research Lab at Stony Brook University and labs in the Department of Biomedical Engineering, particularly to Dr. Wei Yin for assistance in tissue turnover marker analysis, and Alyssa Tuthill for assistance in animal experiment.

\footnotetext{
Authors' roles: This study was conceived by HZK, XL and YXQ. MH performed part of the animal study jointly with DZ. TC was responsible for trabecular bone histomorphometry sectioning and contouring. LL helped with MTS system setting. JW helped with quantification of PINP and TRACP immunoassays. DZ performed animal studies, extraction of tissue samples, $\mu \mathrm{CT}$ analysis, histomorphometry analysis, mechanical bending test and immunoassay analysis. Data was analyzed and interpreted by DZ. The draft manuscript was generated by DZ and revised by each author, particularly by $\mathrm{MH}, \mathrm{XL}$ and YXQ. The draft manuscript was finally approved by YXQ.
} 


\section{References}

[1] Lin C, Jiang X, Dai Z, Guo X, Weng T, Wang J, Li Y, Feng G, Gao X, He L. Sclerostin mediates bone response to mechanical unloading through antagonizing Wnt/beta-catenin signaling. J Bone Miner Res 2009;24: 1651-61.

[2] Li X, Ominsky MS, Warmington KS, Morony S, Gong J, Cao J, Gao Y, Shalhoub V, Tipton B, Haldankar R, Chen Q, Winters A, Boone T, Geng Z, Niu QT, Ke HZ, Kostenuik PJ, Simonet WS, Lacey DL, Paszty C. Sclerostin antibody treatment increases bone formation, bone mass, and bone strength in a rat model of postmenopausal osteoporosis. J Bone Miner Res 2009;24: 578-88.

[3] de Souza RA, Xavier M, da Silva FF, de Souza MT, Tosato MG, Martin AA, Castilho JC, Ribeiro W, Silveira L, Jr. Influence of creatine supplementation on bone quality in the ovariectomized rat model: an FT-Raman spectroscopy study. Lasers Med Sci 2012;27: 487-95.

[4] Sunyecz JA. The use of calcium and vitamin D in the management of osteoporosis. Ther Clin Risk Manag 2008;4: 827-36.

[5] Cauley JA. Public Health Impact of Osteoporosis. J Gerontol A Biol Sci Med Sci 2013.

[6] Becker DJ, Kilgore ML, Morrisey MA. The societal burden of osteoporosis. Curr Rheumatol Rep 2010;12: 186-91.

[7] Burge R, Dawson-Hughes B, Solomon DH, Wong JB, King A, Tosteson A. Incidence and Economic Burden of Osteoporosis-Related Fractures in the United States, 2005-2025. Journal of Bone and Mineral Research 2007;22: 465-475.

[8] Das S, Crockett JC. Osteoporosis - a current view of pharmacological prevention and treatment. Drug Des Devel Ther 2013;7: 435-48.

[9] Bikle DD, Halloran BP. The response of bone to unloading. J Bone Miner Metab 1999;17: 233-44. 
[10] Gaudio A, Pennisi P, Bratengeier C, Torrisi V, Lindner B, Mangiafico RA, Pulvirenti I, Hawa G, Tringali G, Fiore CE. Increased sclerostin serum levels associated with bone formation and resorption markers in patients with immobilization-induced bone loss. J Clin Endocrinol Metab 2010;95: 2248-53.

[11] Shahnazari M, Wronski T, Chu V, Williams A, Leeper A, Stolina M, Ke HZ, Halloran B. Early response of bone marrow osteoprogenitors to skeletal unloading and sclerostin antibody. Calcif Tissue Int 2012;91: 50-8.

[12] Vico L, Chappard D, Alexandre C, Palle S, Minaire P, Riffat G, Morukov B, Rakhmanov S. Effects of a 120 day period of bed-rest on bone mass and bone cell activities in man: attempts at countermeasure. Bone Miner 1987;2: 383-94.

[13] Fiore CE, Pennisi P, Ciffo F, Scebba C, Amico A, Di Fazzio S. Immobilization-dependent bone collagen breakdown appears to increase with time: evidence for a lack of new bone equilibrium in response to reduced load during prolonged bed rest. Horm Metab Res 1999;31: 31-6.

[14] Raisz LG, Prestwood KM. Epidemiology and pathogenesis of osteoporosis. Clin Cornerstone 2000;2: 1-10.

[15] King AC, Rejeski WJ, Buchner DM. Physical activity interventions targeting older adults. A critical review and recommendations. Am J Prev Med 1998;15: 316-33.

[16] Karlsson M. Has exercise an antifracture efficacy in women? Scand J Med Sci Sports 2004;14: 215.

[17] Zernicke R, MacKay C, Lorincz C. Mechanisms of bone remodeling during weight-bearing exercise. Appl Physiol Nutr Metab 2006;31: 655-60.

[18] Tou JC, Foley A, Yuan YV, Arnaud S, Wade CE, Brown M. The effect of ovariectomy combined with hindlimb unloading and reloading on the long bones of mature Sprague-Dawley rats. Menopause 2008;15: 494-502. 
[19] Kim BJ, Bae SJ, Lee SY, Lee YS, Baek JE, Park SY, Lee SH, Koh JM, Kim GS. TNF-alpha mediates the stimulation of sclerostin expression in an estrogen-deficient condition. Biochem Biophys Res Commun 2012;424: 170-5.

[20] Li X, Zhang Y, Kang H, Liu W, Liu P, Zhang J, Harris SE, Wu D. Sclerostin binds to LRP5/6 and antagonizes canonical Wnt signaling. J Biol Chem 2005;280: 19883-7.

[21] Winkler DG, Sutherland MK, Geoghegan JC, Yu C, Hayes T, Skonier JE, Shpektor D, Jonas M, Kovacevich BR, Staehling-Hampton K, Appleby M, Brunkow ME, Latham JA. Osteocyte control of bone formation via sclerostin, a novel BMP antagonist. EMBO J 2003;22: 6267-76.

[22] Rossini M, Gatti D, Adami S. Involvement of WNT/beta-catenin Signaling in the Treatment of Osteoporosis. Calcif Tissue Int 2013;93: 121-32.

[23] Spatz JM, Fields EE, Yu EW, Divieti Pajevic P, Bouxsein ML, Sibonga JD, Zwart SR, Smith SM. Serum sclerostin increases in healthy adult men during bed rest. J Clin Endocrinol Metab 2012;97: E1736-40.

[24] Lewiecki EM. Sclerostin: a novel target for intervention in the treatment of osteoporosis. Discov Med 2011;12: 263-73.

[25] Qin L, Ding DX, Cui LL, Huang QY. [Expression and regulation of the SOST gene]. Yi Chuan 2013;35: 939-47.

[26] van Dinther M, Zhang J, Weidauer SE, Boschert V, Muth EM, Knappik A, de Gorter DJ, van Kasteren PB, Frisch C, Mueller TD, ten Dijke P. Anti-Sclerostin antibody inhibits internalization of Sclerostin and Sclerostin-mediated antagonism of Wnt/LRP6 signaling. PLoS One 2013;8: e62295.

[27] Li X, Ominsky MS, Niu QT, Sun N, Daugherty B, D'Agostin D, Kurahara C, Gao Y, Cao J, Gong J, Asuncion F, Barrero M, Warmington K, Dwyer D, Stolina M, Morony S, Sarosi I, Kostenuik PJ, Lacey DL, Simonet WS, Ke HZ, Paszty C. Targeted deletion of the sclerostin gene in mice results in increased bone formation and bone strength. J Bone Miner Res 2008;23: 860-9. 
[28] Padhi D, Jang G, Stouch B, Fang L, Posvar E. Single-dose, placebo-controlled, randomized study of AMG 785, a sclerostin monoclonal antibody. J Bone Miner Res 2011;26: 19-26.

[29] Poole KE, van Bezooijen RL, Loveridge N, Hamersma H, Papapoulos SE, Lowik CW, Reeve J. Sclerostin is a delayed secreted product of osteocytes that inhibits bone formation. FASEB J 2005;19: 1842-4.

[30] van Bezooijen RL, ten Dijke P, Papapoulos SE, Lowik CW. SOST/sclerostin, an osteocyte-derived negative regulator of bone formation. Cytokine Growth Factor Rev 2005;16: 319-27.

[31] Tian X, Jee WS, Li X, Paszty C, Ke HZ. Sclerostin antibody increases bone mass by stimulating bone formation and inhibiting bone resorption in a hindlimb-immobilization rat model. Bone 2011;48: 197-201.

[32] Lam H, Qin YX. The effects of frequency-dependent dynamic muscle stimulation on inhibition of trabecular bone loss in a disuse model. Bone 2008;43: 1093-100.

[33] Hu M, Cheng J, Qin YX. Dynamic hydraulic flow stimulation on mitigation of trabecular bone loss in a rat functional disuse model. Bone 2012;51: 819-25.

[34] Chen H, Xu X, Liu M, Zhang W, Ke HZ, Qin A, Tang T, Lu E. Sclerostin antibody treatment causes greater alveolar crest height and bone mass in an ovariectomized rat model of localized periodontitis. Bone 2015;76: 141-8.

[35] Stolina M, Dwyer D, Niu QT, Villasenor KS, Kurimoto P, Grisanti M, Han CY, Liu M, Li X, Ominsky MS, Ke HZ, Kostenuik PJ. Temporal changes in systemic and local expression of bone turnover markers during six months of sclerostin antibody administration to ovariectomized rats. Bone 2014;67: 305-13.

[36] Korres N, Tsiridis E, Pavlou G, Mitsoudis A, Perrea DN, Zoumbos AB. Biomechanical characteristics of bone in streptozotocin-induced diabetic rats: An in-vivo randomized controlled experimental study. World J Orthop 2013;4: 124-9. 
[37] Turner CH, Burr DB. Basic biomechanical measurements of bone: a tutorial. Bone 1993;14: 595608.

[38] Spatz JM, Ellman R, Cloutier AM, Louis L, van Vliet M, Suva L, Dwyer D, Stolina M, Ke HZ, Bouxsein ML. Sclerostin antibody inhibits skeletal deterioration due to reduced mechanical loading. J Bone Miner Res 2013;28: 865-74.

[39] Melton L, 3rd, Christen D, Riggs BL, Achenbach SJ, Muller R, van Lenthe GH, Amin S, Atkinson EJ, Khosla S. Assessing forearm fracture risk in postmenopausal women. Osteoporos Int 2010;21: 1161-9. [40] Hildebrand T, Ruegsegger P. Quantification of Bone Microarchitecture with the Structure Model Index. Comput Methods Biomech Biomed Engin 1997;1: 15-23.

[41] Li X, Warmington KS, Niu QT, Asuncion FJ, Barrero M, Grisanti M, Dwyer D, Stouch B, Thway TM, Stolina M, Ominsky MS, Kostenuik PJ, Simonet WS, Paszty C, Ke HZ. Inhibition of sclerostin by monoclonal antibody increases bone formation, bone mass, and bone strength in aged male rats. J Bone Miner Res 2010;25: 2647-56.

[42] Janckila AJ, Parthasarathy RN, Parthasarathy LK, Seelan RS, Hsueh YC, Rissanen J, Alatalo SL, Halleen JM, Yam LT. Properties and expression of human tartrate-resistant acid phosphatase isoform 5a by monocyte-derived cells. J Leukoc Biol 2005;77: 209-18.

[43] Rissanen JP, Suominen MI, Peng Z, Halleen JM. Secreted tartrate-resistant acid phosphatase 5b is a Marker of osteoclast number in human osteoclast cultures and the rat ovariectomy model. Calcif Tissue Int 2008;82: 108-15.

[44] Alatalo SL, Halleen JM, Hentunen TA, Monkkonen J, Vaananen HK. Rapid screening method for osteoclast differentiation in vitro that measures tartrate-resistant acid phosphatase $5 \mathrm{~b}$ activity secreted into the culture medium. Clin Chem 2000;46: 1751-4.

[45] Li X, Ominsky MS, Warmington KS, Niu QT, Asuncion FJ, Barrero M, Dwyer D, Grisanti M, Stolina M, Kostenuik PJ, Simonet WS, Paszty C, Ke HZ. Increased bone formation and bone mass induced by 
sclerostin antibody is not affected by pretreatment or cotreatment with alendronate in osteopenic, ovariectomized rats. Endocrinology 2011;152: 3312-22.

[46] Sun X, Liang J, Wang C, Cao S, Hu Y, Xu X. Transient Effect of 17beta-estradiol on Osteoporosis in Ovariectomized Rats Accompanied with Unilateral Disuse in the Early Phase. Int J Med Sci 2015;12: 42331.

[47] Tatsumi S, Ishii K, Amizuka N, Li M, Kobayashi T, Kohno K, Ito M, Takeshita S, Ikeda K. Targeted ablation of osteocytes induces osteoporosis with defective mechanotransduction. Cell Metab 2007;5: 464-75.

[48] Glass li DA, Bialek P, Ahn JD, Starbuck M, Patel MS, Clevers H, Taketo MM, Long F, McMahon AP, Lang RA, Karsenty G. Canonical Wnt Signaling in Differentiated Osteoblasts Controls Osteoclast Differentiation. Developmental Cell 2005;8: 751-764.

[49] Takahashi N, Maeda K, Ishihara A, Uehara S, Kobayashi Y. Regulatory mechanism of osteoclastogenesis by RANKL and Wnt signals. Front Biosci (Landmark Ed) 2011;16: 21-30.

[50] Tu X, Rhee Y, Condon KW, Bivi N, Allen MR, Dwyer D, Stolina M, Turner CH, Robling AG, Plotkin LI, Bellido T. Sost downregulation and local Wnt signaling are required for the osteogenic response to mechanical loading. Bone 2012;50: 209-17.

[51] van Bezooijen RL, Roelen BA, Visser A, van der Wee-Pals L, de Wilt E, Karperien M, Hamersma H, Papapoulos SE, ten Dijke P, Lowik CW. Sclerostin is an osteocyte-expressed negative regulator of bone formation, but not a classical BMP antagonist. J Exp Med 2004;199: 805-14.

[52] Glass DA, 2nd, Karsenty G. In vivo analysis of Wnt signaling in bone. Endocrinology 2007;148: 2630-4.

[53] Baron R, Rawadi G. Targeting the Wnt/beta-catenin pathway to regulate bone formation in the adult skeleton. Endocrinology 2007;148: 2635-43. 
[54] Mirza FS, Padhi ID, Raisz LG, Lorenzo JA. Serum sclerostin levels negatively correlate with parathyroid hormone levels and free estrogen index in postmenopausal women. J Clin Endocrinol Metab 2010;95: 1991-7.

[55] Cejka D, Herberth J, Branscum AJ, Fardo DW, Monier-Faugere MC, Diarra D, Haas M, Malluche HH. Sclerostin and Dickkopf-1 in renal osteodystrophy. Clin J Am Soc Nephrol 2011;6: 877-82. 


\section{Figure Caption}

Figure 1. a) $\mu \mathrm{CT}$ reconstruction images of trabecular area of distal femur of rats. b) Graphs show mean+SD values for bone volume fraction (BV/TV, \%). c) Mean+SD values for trabecular thickness (Tb.Th, $\mu \mathrm{m})$. d) Mean+SD values for SMI. ${ }^{*} \mathrm{p}<0.05$ vs. Sham+Veh; $\stackrel{\wedge}{\mathrm{p}}<0.05$ vs. Sham+HLS+Veh; ${ }^{\#}$ p<0.05 vs. OVX+Veh; ${ }^{\&}$ p<0.05 vs. OVX+HLS+Veh.

Figure 2. a) Mean+SD values for 4-point bending stiffness. b) Mean+SD values for 4-point bending ultimate load. ${ }^{*} \mathrm{p}<0.05$ vs. Sham+Veh; ${ }^{\wedge} \mathrm{p}<0.05$ vs. Sham+HLS+Veh; ${ }^{\#} \mathrm{p}<0.05$ vs. OVX+Veh; ${ }^{\&} \mathrm{p}<0.05$ vs. OVX+HLS+Veh.

Figure 3. Mean+SD of histomorphometric analysis of distal femoral metaphysis trabecular bone. Graphs a)-e) shows Mean+SD values for 2D bone volume fraction (aBV/TV, \%), 2D trabecular thickness (Tb.Th, $\mu \mathrm{m})$, mineral surface (MS/BS, \%), mineral apposition rate (MAR, $\mu \mathrm{m} /$ day) and bone formation rate (BFR/BS, $\mu \mathrm{m} 3 / \mu \mathrm{m} 2 /$ day). f) Representative images of calcein labeled

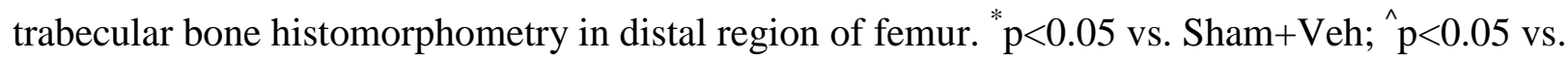
Sham+HLS+Veh; ${ }^{\#}$ p $<0.05$ vs. OVX+Veh; ${ }^{\&}$ p $<0.05$ vs. OVX+HLS+Veh.

Figure 4. Mean+SD value of TRACP $5 b$ level normalized to age-match control level at sacrifice. $\hat{p}<0.05$ vs. Sham + HLS + Veh 


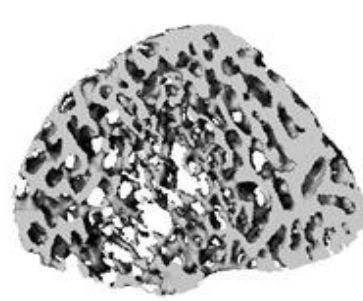

Sham+Veh

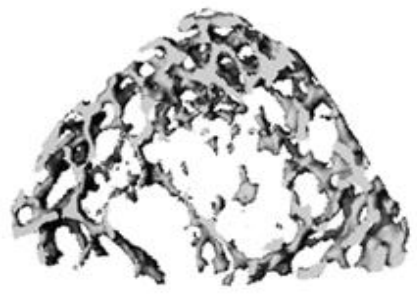

Sham+HLS+Veh

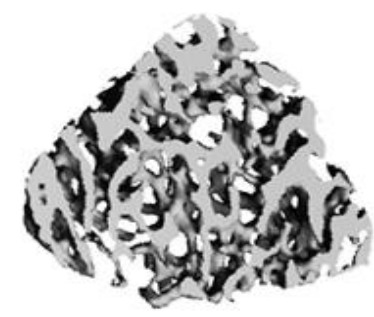

Sham $+\mathrm{HLS}+\mathrm{Scl}-\mathrm{Ab}$

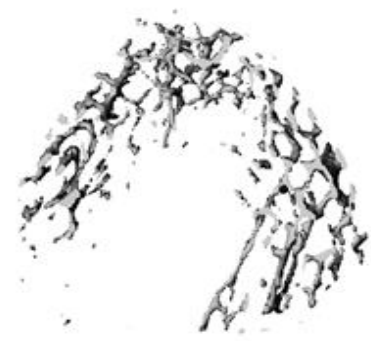

OVX+Veh

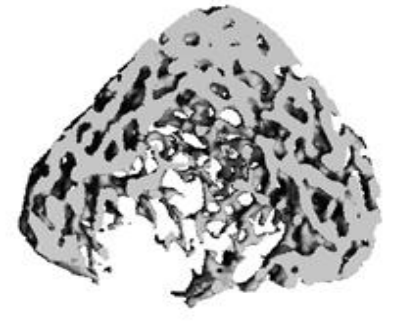

$\mathrm{OVX}+\mathrm{Scl}-\mathrm{Ab}$

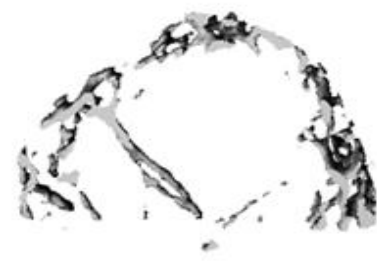

$\mathrm{OVX}+\mathrm{HLS}+\mathrm{Veh}$

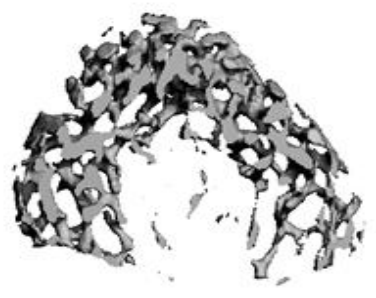

$\mathrm{OVX}+\mathrm{HLS}+\mathrm{Scl}-\mathrm{Ab}$

Fig. 1(a) 


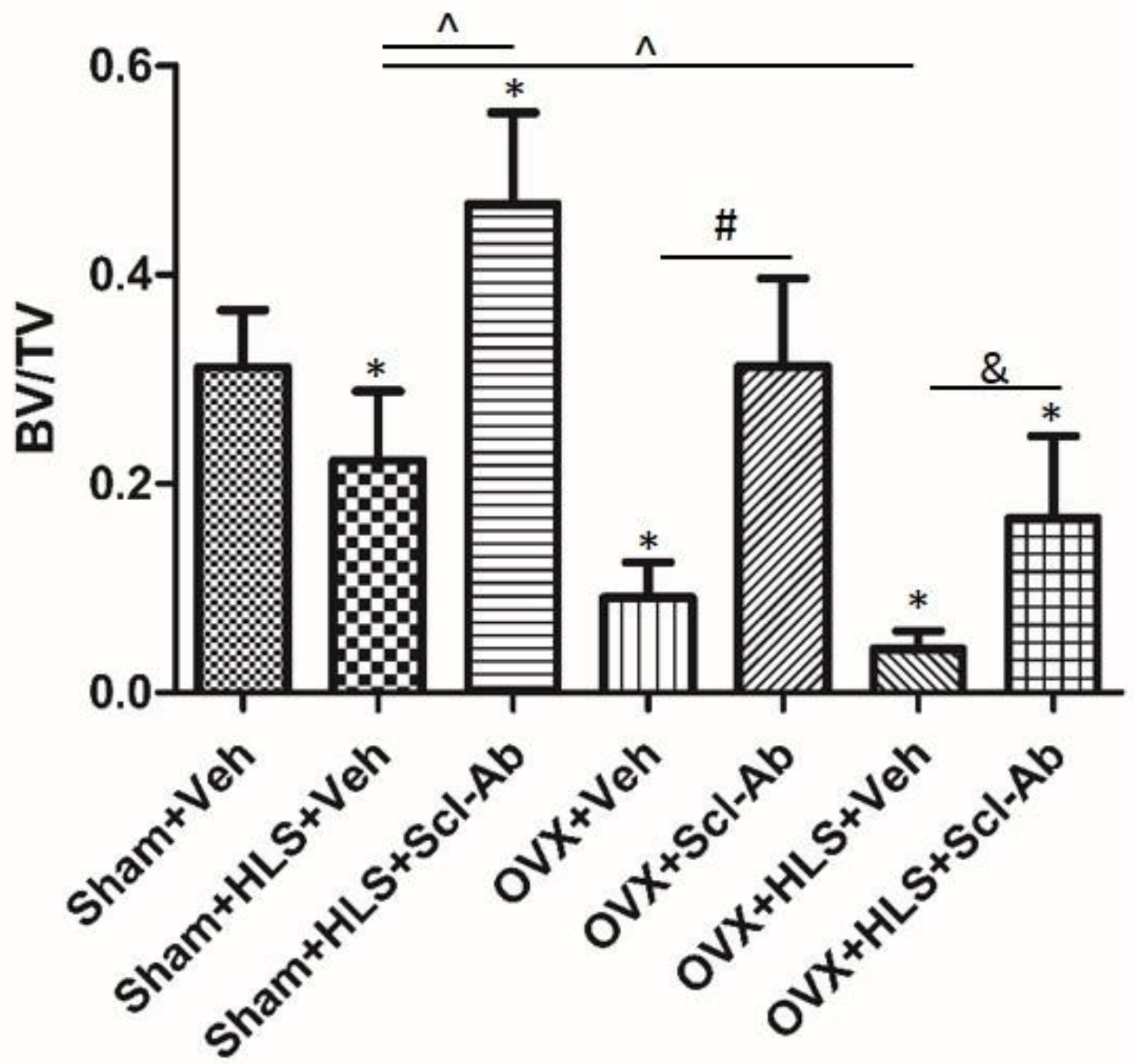

Fig. 1(b) 


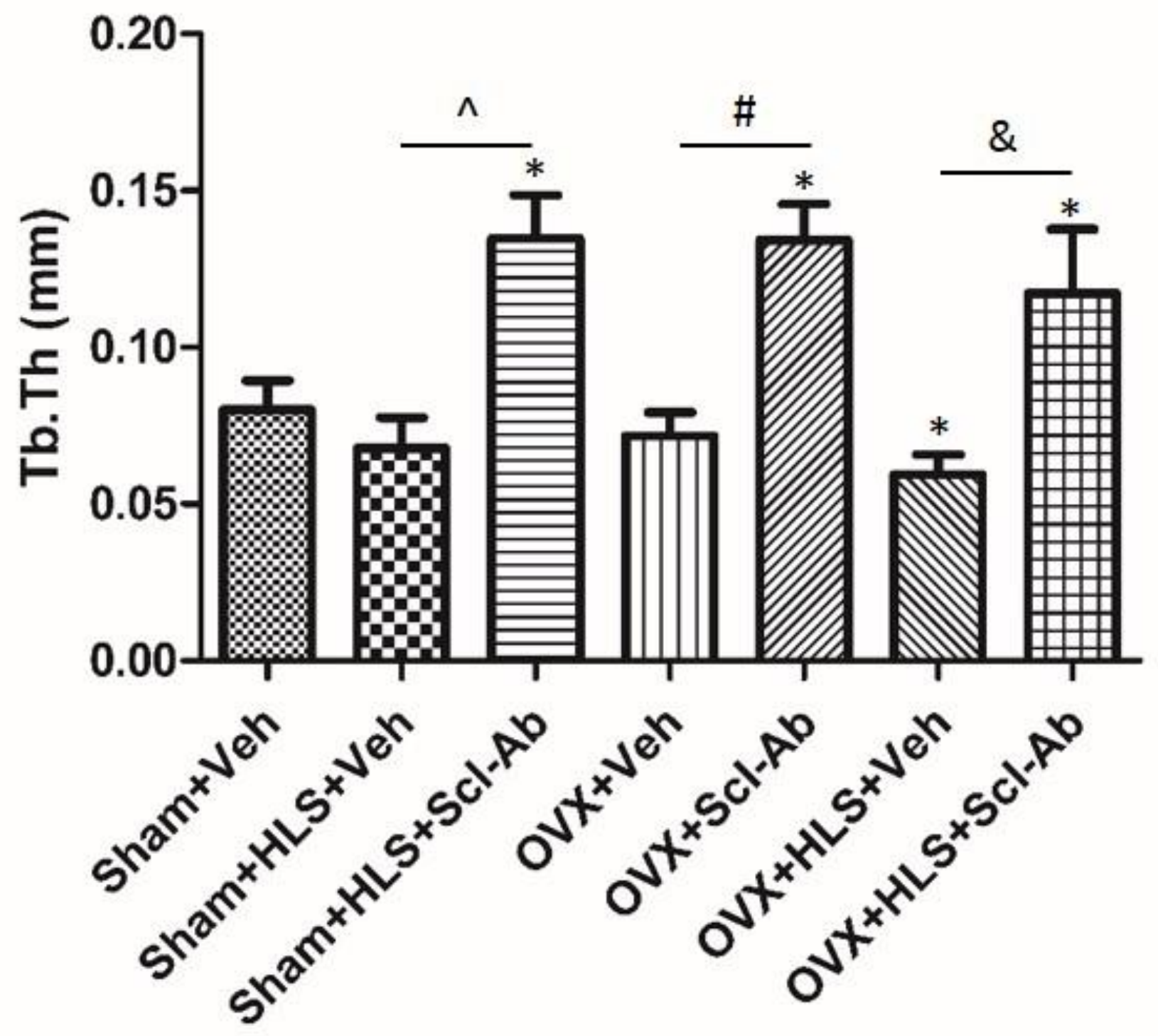

Fig. 1(c) 

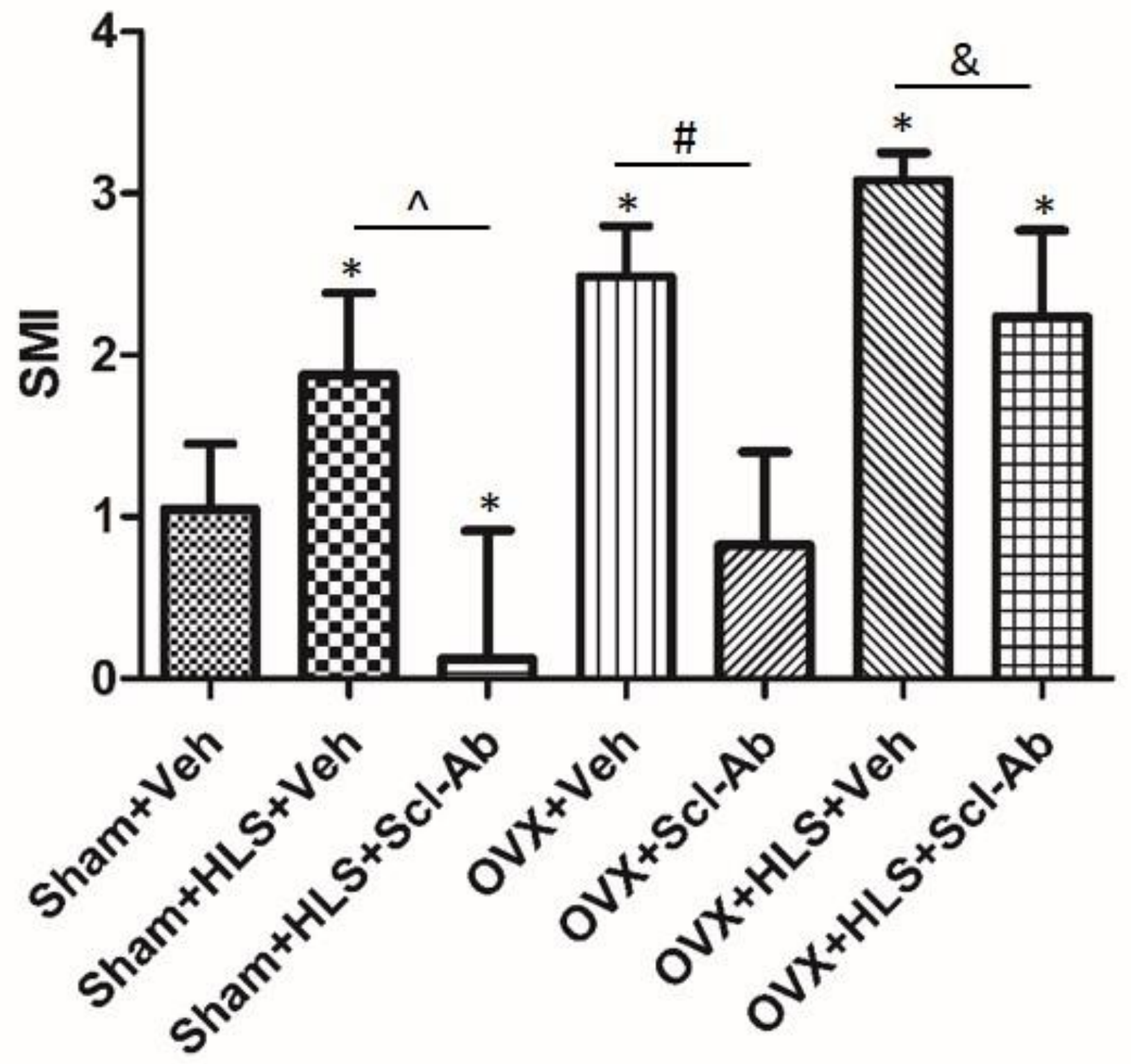

Fig. 1(d) 


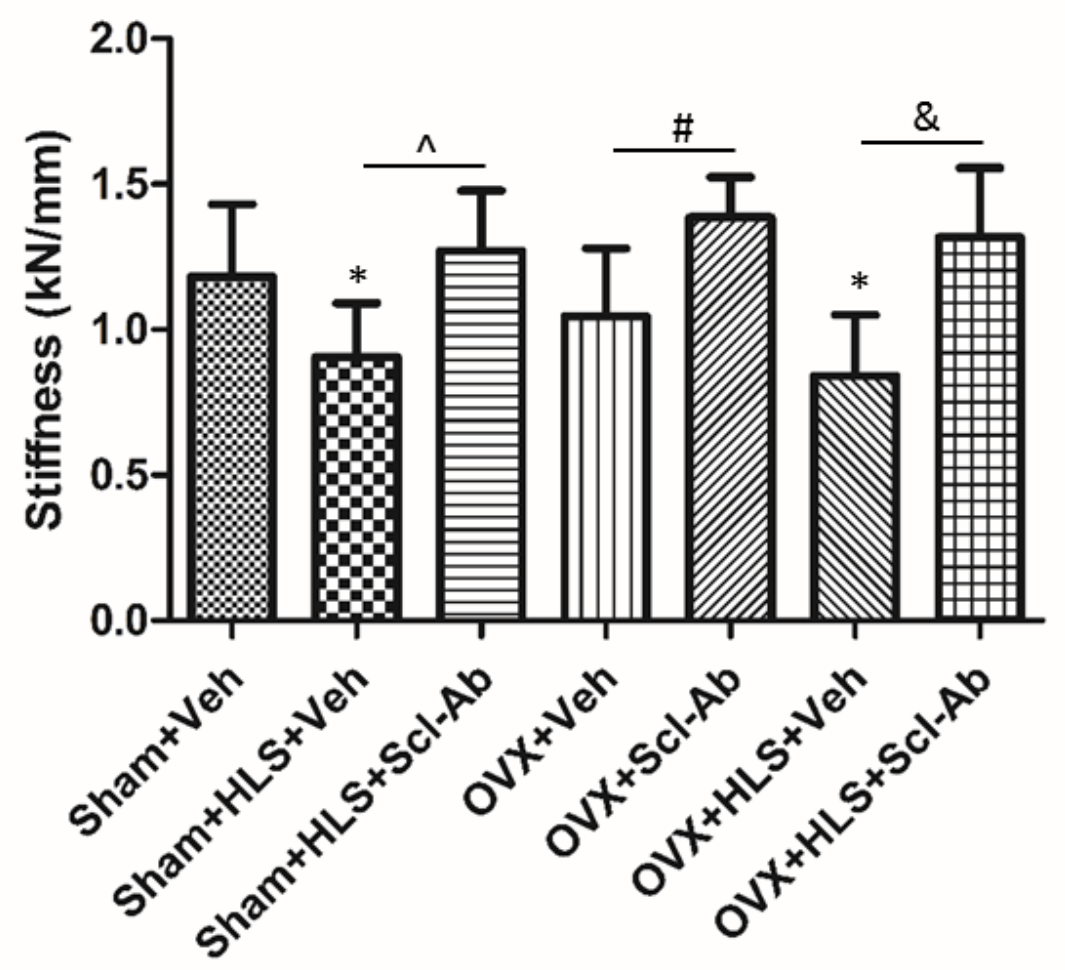

Fig. 2(a)

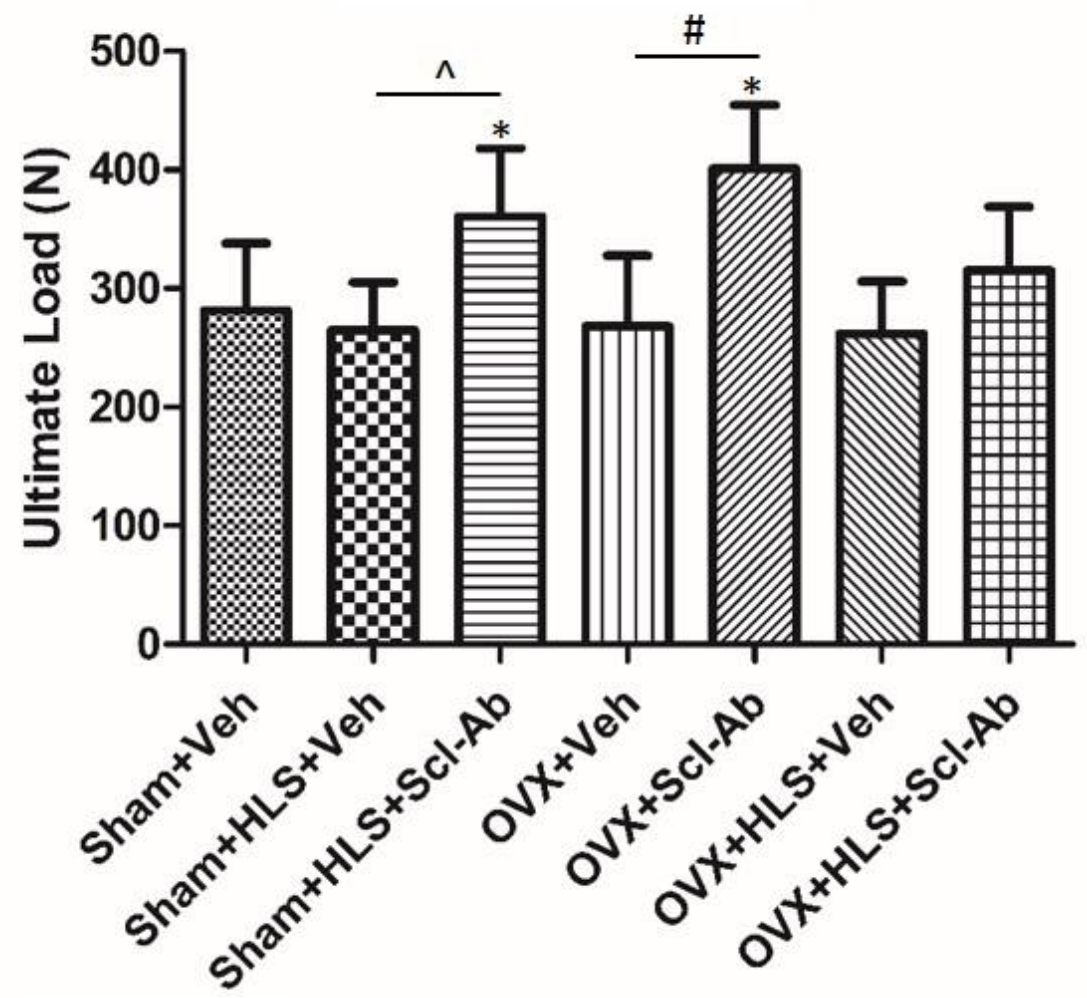

Fig. 2(b) 


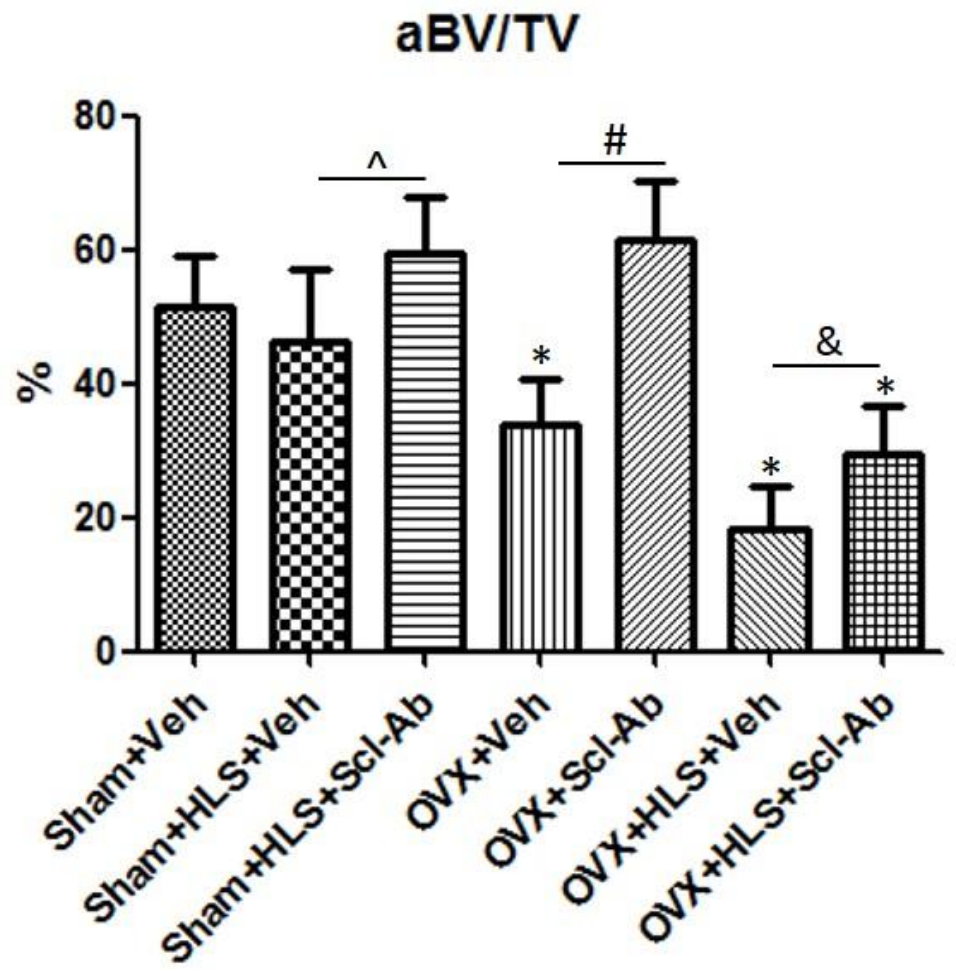

Fig. 3(a)

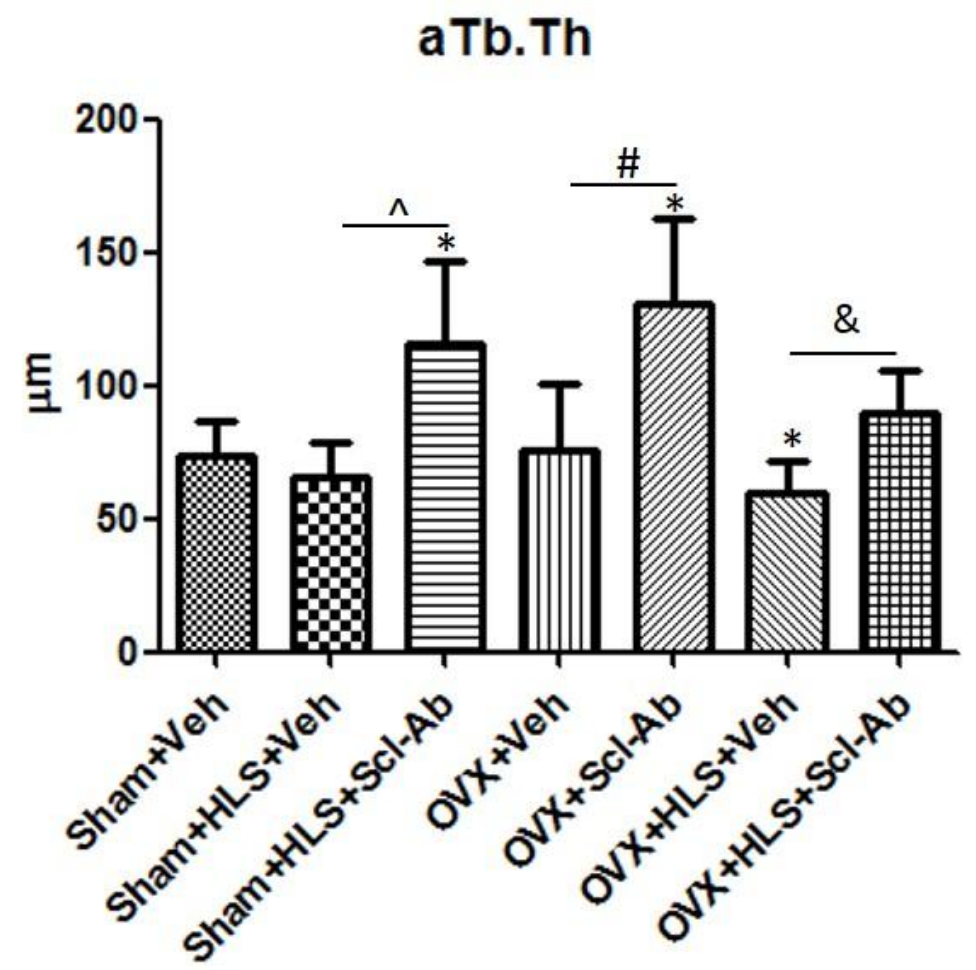

Fig. 3(b) 
MS/BS

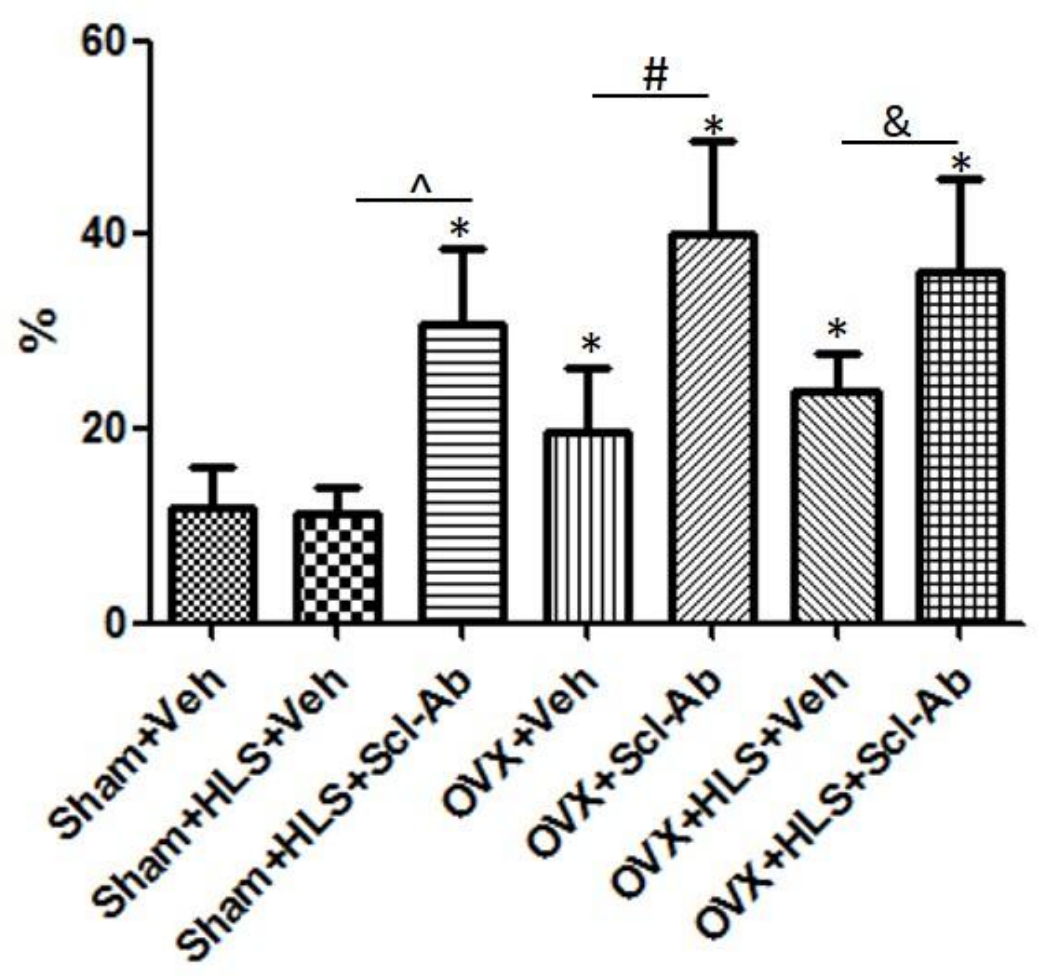

Fig. 3(c)

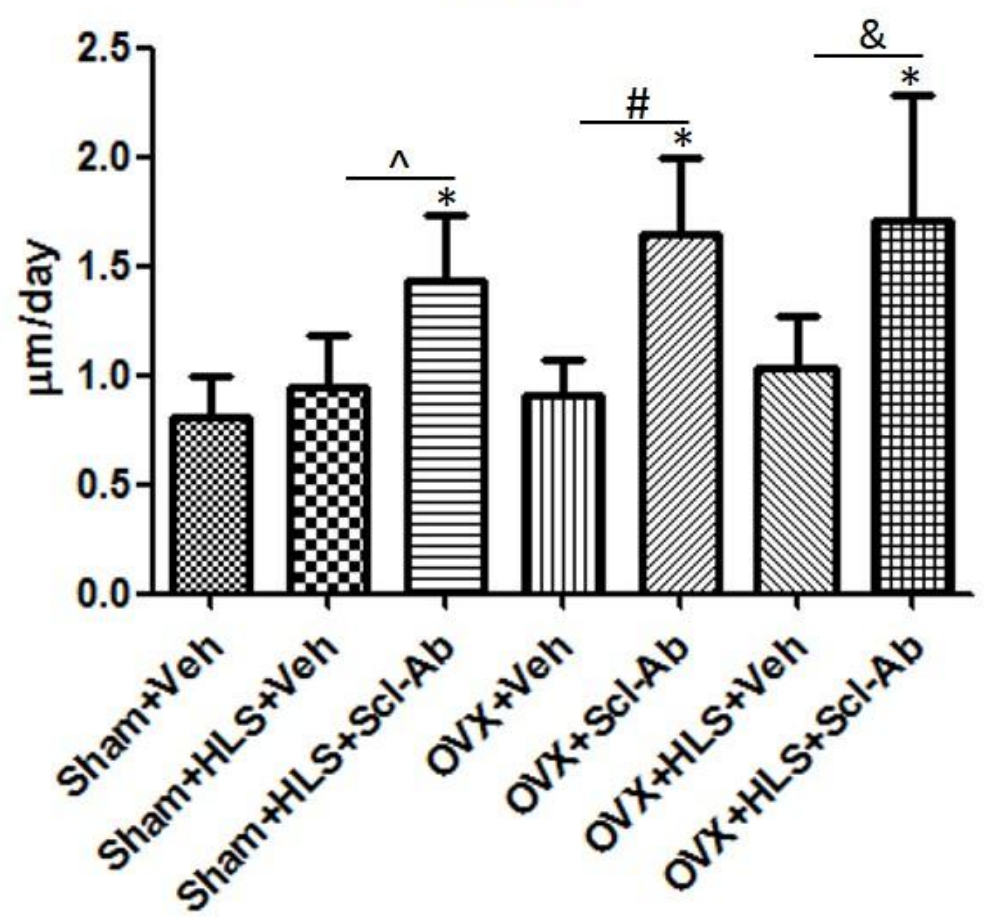

Fig. 3(d) 


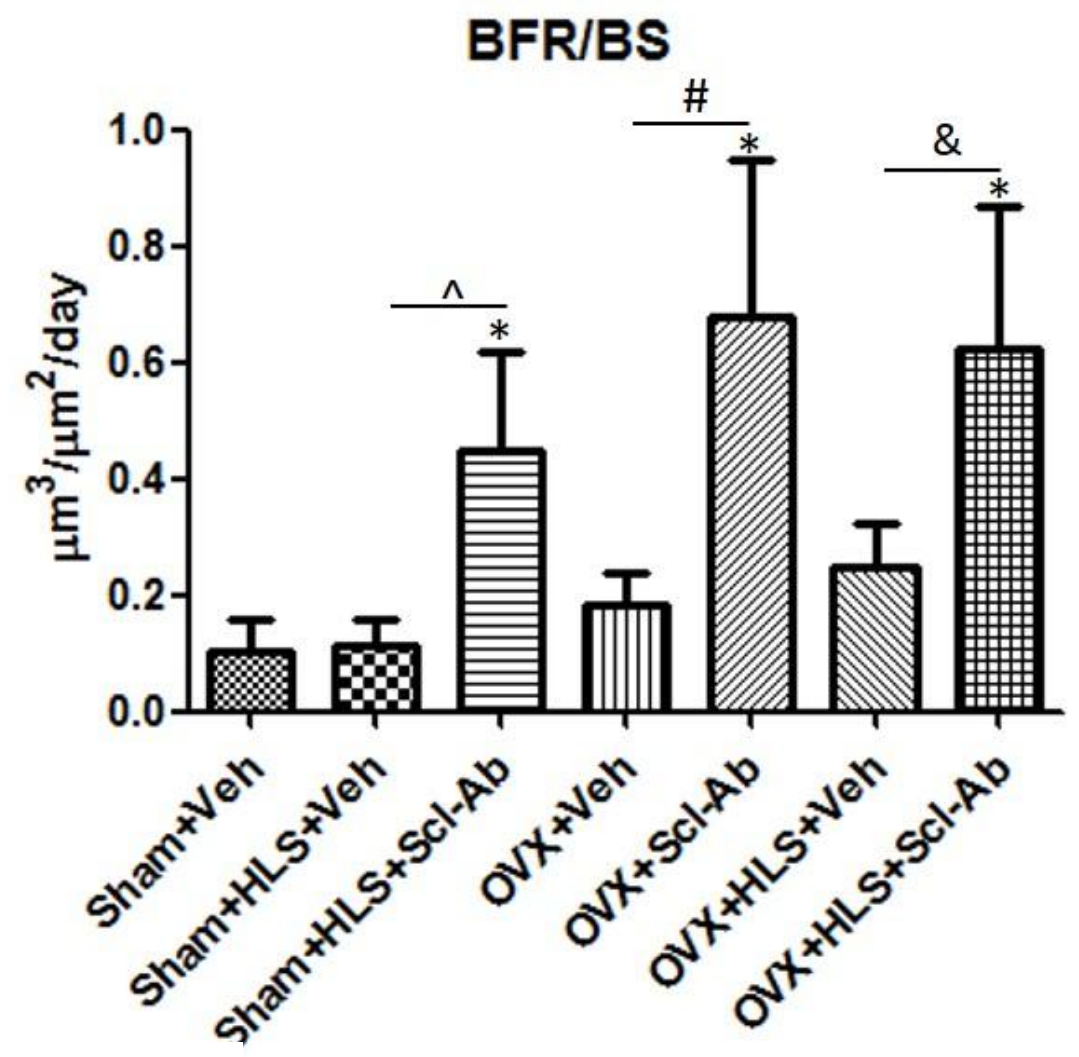

Fig. 3(e) 
Figure $3 \mathrm{f}$
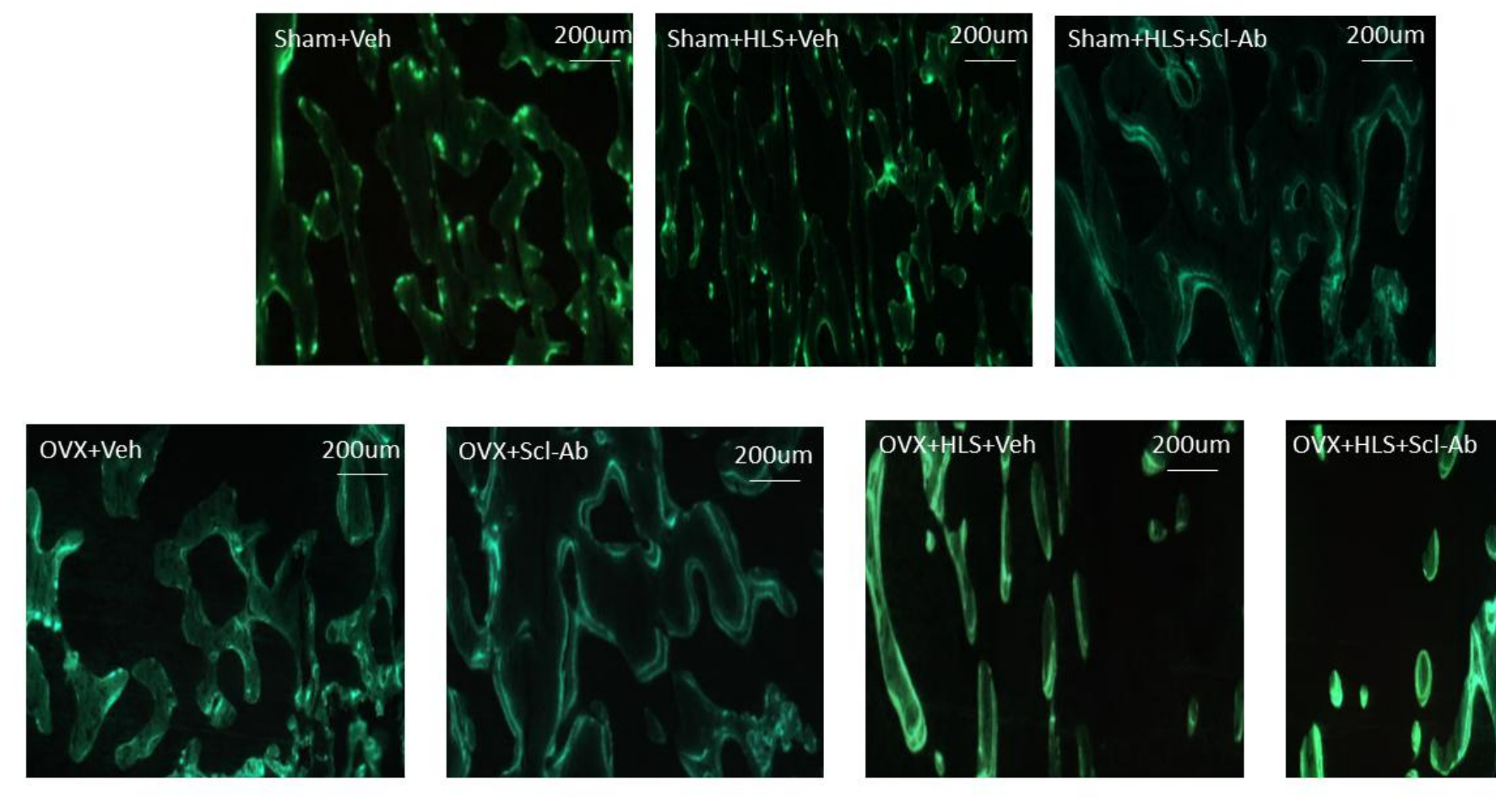

Fig. 3(f)

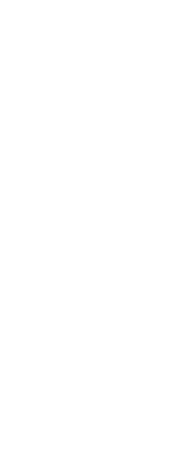

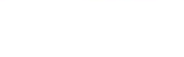

\section{Fig. $3(f)$}
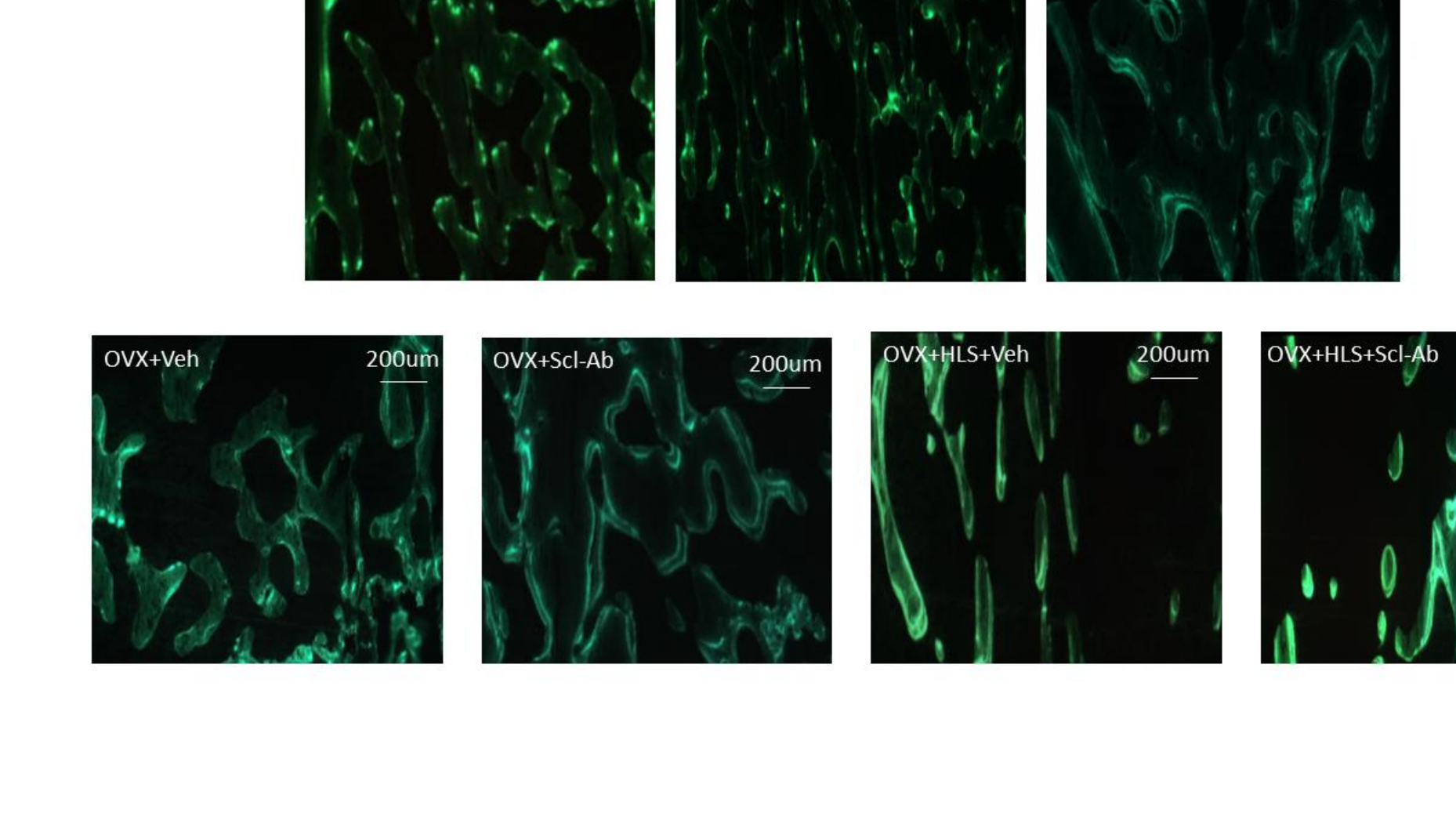
Figure 4

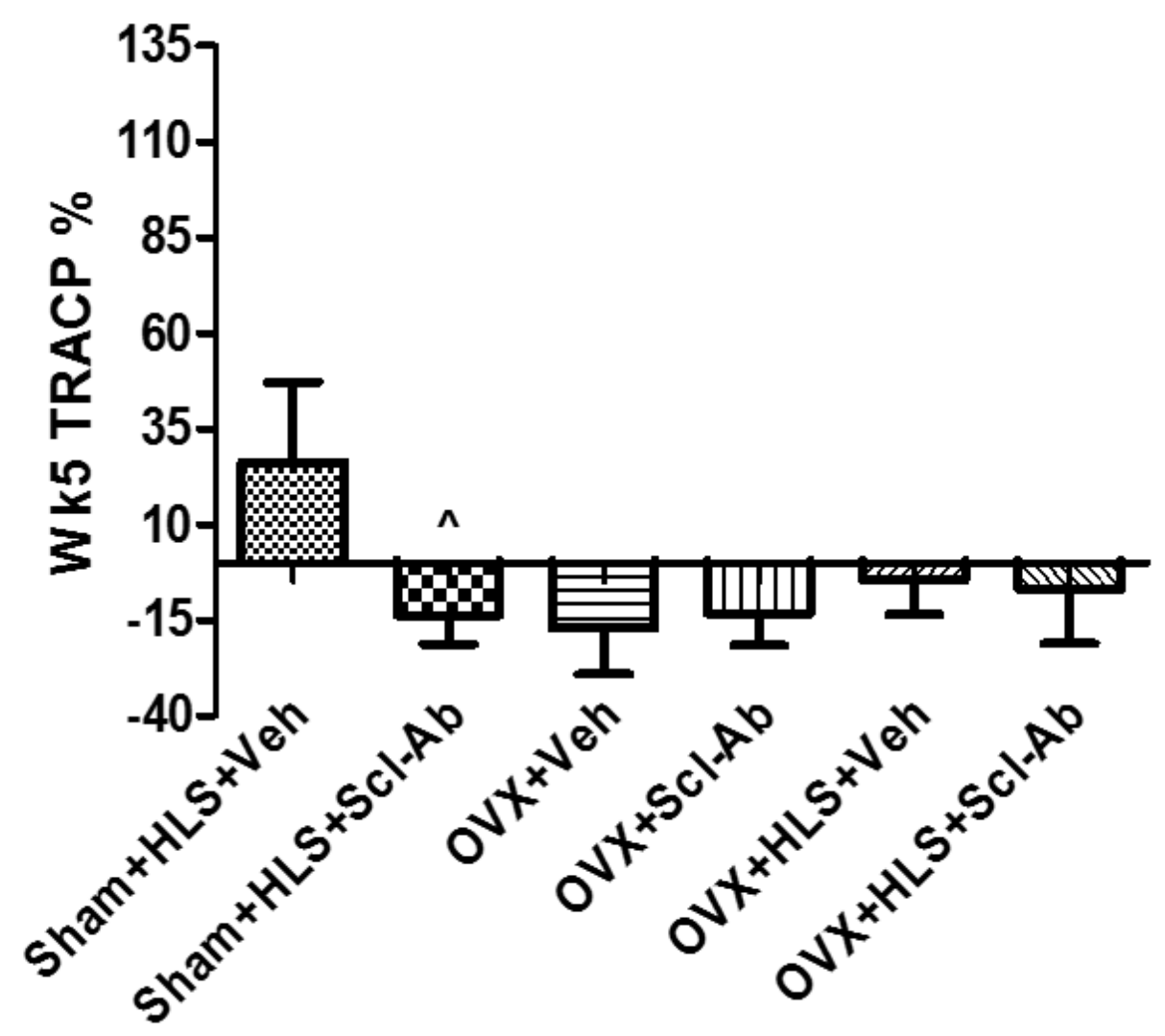

Fig. 4 